An isotropic model for cluster growth on a regular lattice

\author{
Christian A. Yates and Ruth E. Baker \\ Centre for Mathematical Biology, Mathematical Institute, \\ University of Oxford, 24-29 St Giles', Oxford OX1 3LB, UK*
}

(Dated: June 11, 2013)

Abstract

*yatesc@maths.ox.ac.uk; http://people.maths.ox.ac.uk/yatesc/ 
On-lattice models for cluster aggregation have been studied for at least the last 50 years. One of the earliest, originally designed to simulate tumor formation, is known as the Eden model $[12,13]$ and comes in three versions $\mathrm{A}, \mathrm{B}$ and $\mathrm{C}[19]$. In each version particles are added to the surface of the cluster incrementally. On-lattice cluster aggregation models like Eden's have been used to represent biological phenomena such as wound healing [10], slime mold growth [26] and, more recently, the in vitro growth of tumor monolayers [8, 11].

Some models of cluster aggregation can be classified into universality classes (UCs) depending on the surface scaling properties of their aggregates. For example, version $\mathrm{C}$ of the Eden model has scaling characteristics which are most similar to those of the Kardar-ParisiZhang (KPZ) UC [7, 19]. The continuum equation associated with the KPZ UC predicts circular clusters [7]. However, when Eden clusters are realized on a regular lattice there is an inherent anisotropy which does not diminish as the clusters grow larger [6] (see Fig. 1 (a) of the supplementary material (SM)); the shape of large on-lattice Eden clusters is dominated by the underlying lattice anisotropy (see SM Fig. 1 (b)). Such artificial anisotropy is clearly an undesirable property for any on-lattice cluster growth model.

Several related models have attempted to reduce the effects of this inherent, regularlattice-induced anisotropy, by altering or completely revising the cluster aggregation algorithms. However, few of these attempts have been shown to produce a truly isotropic cluster aggregation model. In this paper we adapt and analyse a little known cluster formation model, 'Model II' of Ferreira Jr. and Alves [15], so that cluster growth is driven by random elemental division and rearrangement events on a square lattice. We demonstrate the isotropy of the model in both the noisy and noise-reduced limits by considering both a bespoke anisotropy measure and a more-standard series decomposition of the surface. The elemental division mechanism we introduce can also be thought of as more akin to biological growth than the surface addition rules employed by many Eden-derived models of cluster aggregation. The clusters produced also have the desirable properties of being concave and hole-free. We go on to investigate the scaling properties of this aggregation model and find that the traditional techniques of classification into UCs are not sufficient for this model. A new surface scaling relationship which may be more appropriate for this cluster aggregation model is suggested. We conclude by employing the revised model with the explicit elemental division algorithm to act as the lattice upon which a position-jump model of diffusion is implemented. This model of diffusion on a growing domain is compared both qualitatively 
and quantitatively to a continuum model of the same process with good agreement being found in the mean field.

\section{MEASURING ANISOTROPY}

In order to accurately determine the anisotropy of a cluster it is important to have a method of measurement which is itself free of lattice artifacts. Although box-counting methods such as those employed by Meakin [21] can be useful in characterizing large-scale anisotropies they may suffer from anisotropy themselves. As such we consider a bespoke measure, the 'angular surface anisotropy'. This is given by the normalized square of the distance to the surface from the center of the cluster in a particular direction $[6,16]$ : for each surface element, $i$, calculate the angle, $\phi_{i}$, subtended by the $x$-axis and the line connecting the center of the seed particle (or center of mass, translating the cluster so that this lies at the origin) to the element's center (see Fig. 1). Also calculate the element's distance from the seed/center of mass, $r_{i}$ (see Fig. 1). The angular surface anisotropy in the direction $\theta_{k}$ is then given by

$$
P\left(\theta_{k}\right)=\frac{1}{N_{s}^{\theta_{k}}} \sum_{i=1}^{N_{s}} r_{i}^{2} \mathbb{I}_{\theta_{k}-\delta \theta<\phi_{i}<\theta_{k}+\delta \theta}
$$

where the sum is over the $N_{s}^{\theta_{k}}=\sum_{i=1}^{N_{s}} \mathbb{I}_{\theta_{k}-\delta \theta<\phi_{i}<\theta_{k}+\delta \theta}$ surface elements with angles in the appropriate region $\left[\theta_{k}-\delta \theta, \theta_{k}+\delta \theta\right]$ (green stars in Fig. 1). II represents the indicator function. The $K$ values of $\theta_{k}$ are chosen so as to cover the entire circle. This necessarily defines $\delta \theta=\pi / K$. We choose $K$ to be a power of two [1] and $\theta_{1}=0$ in order to capture the anisotropy that might be caused by the square lattice. The angular surface anisotropy is normalized by dividing $P\left(\theta_{k}\right)$ by $\sum_{k=1}^{K} P\left(\theta_{k}\right)$ for each value of $k$.

In order to quantitatively analyse the angular surface anisotropy, $P(\theta)$, we can consider a Fourier decomposition of the curve. Fourier modes with large coefficients will indicate larger contributions to the overall anisotropy from that mode. For example, for a cluster with positive axial anisotropy we would expect to see a large positive quadrupole (coefficient of the fourth Fourier mode). 


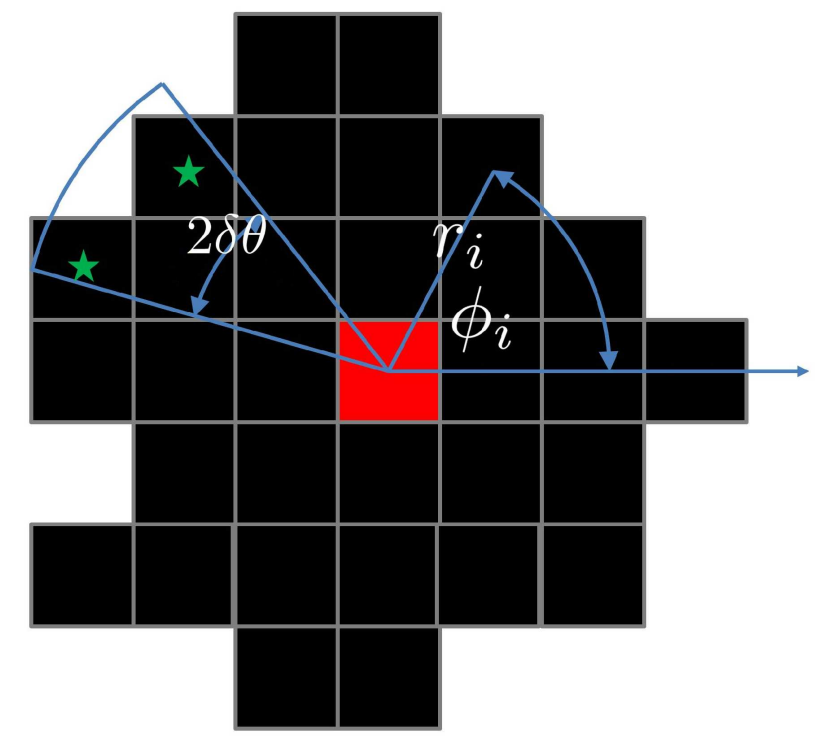

FIG. 1. Measuring anisotropy of particle aggregates. In this example cluster elements of the aggregate are represented by black squares (-). The angle to the $x$-axis, $\phi_{i}$, and the distance from the seed particle (red square, $\mathbf{\square}), r_{i}$, are determined for each element. In each segment of the circle the mean square distance of the centers of mass of the surface elements (green stars, $\star$ ) is calculated and then normalized to find the 'angular surface anisotropy'.

\section{SOME ANISOTROPIC MODELS}

In order to get a better idea of the anisotropy of small clusters 'noise reduction' can be employed [6]. Noise reduction is a useful tool for reducing finite size effects which can sometimes obscure inherent anisotropy in small clusters. (See the SM for a detailed description.) The Eden model exhibits anisotropy in both the noisy and noise-reduced limits (see SM Figs. 1 and 2, respectively).

There have, however, been several Eden-like cluster aggregation models which purport to produce isotropic clusters. Paiva and Ferreira Jr [23], for example, have found on-lattice versions of the Eden $\mathrm{A}$ and $\mathrm{C}$ models which are isotropic (see Fig. 2). Growth events, in these models, depend on the neighborhood of the free space into which a new element is to be positioned. Their technique for ensuring anisotropy is inspired by an isotropic, on-lattice version of diffusion limited aggregation (DLA) by Bogoyavlenskiy [9]. In Bogoyavlenskiy's model, growth at a selected site is implemented with probability $P_{i}=i^{2}$ where $i$ is the number of occupied nearest-neighbors of the growth site. However, this choice of occupancydependent probability was found to produce diagonal anisotropy in the DLA clusters [3]. 
Instead Alves and Ferreira Jr. [3] suggested growth probabilities given by

$$
P_{k}=\left(\frac{i}{n}\right)^{\nu}
$$

where $n$ is the lattice coordination number (i.e. number of possible neighbors of a lattice site), $\nu$ is an adjustable parameter and $i$, as before, is the number of occupied nearest-neighbors of a site selected for growth. The majority of the work in this method is finding the value of the tunable parameter, $\nu$, which minimises the anisotropy. Paiva and Ferreira Jr [23] do this by recognizing that the scaling behavior of the Eden model is affected by the lattice anisotropy. They reason that if they can find an exponent $\nu=\nu_{c}$, in equation (2), for which the growth parameter, $\beta$, of the on-lattice model is as close as possible to its predicted value, $1 / 3$, then this will be the value that produces the most isotropic clusters. Using this method they find $\nu_{c} \approx 1$ for version $\mathrm{C}$ of the Eden model and $\nu_{c} \approx 1.72$ for version $\mathrm{A}$ of the model, although they do not provide any quantitative comparisons of the clusters' anisotropy for these values other than visually comparing the cluster border with a circle.

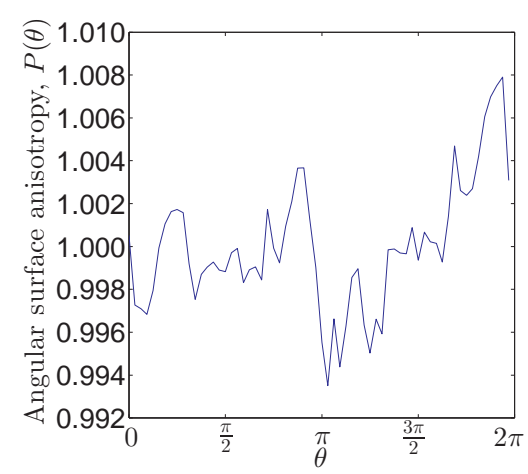

(a)

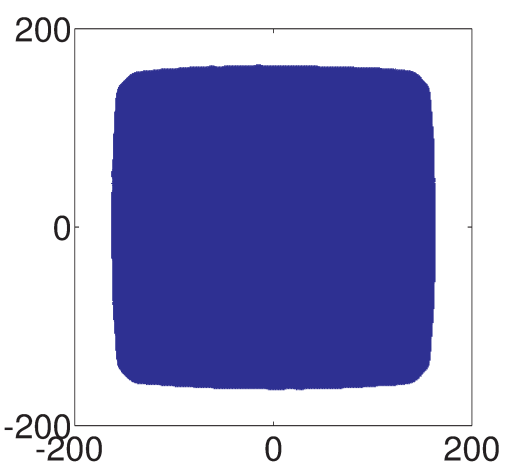

(b)

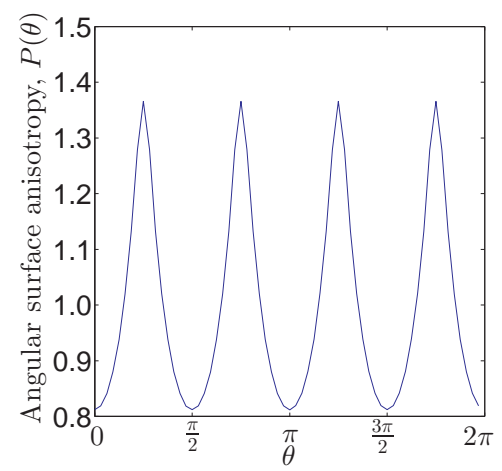

(c)

FIG. 2. The anisotropy of the 'isotropic' Eden C model of Paiva and Ferreira Jr [23]. (a) The angular surface anisotropy, $P(\theta)$, of the 'isotropic' Eden $C$ model displays no clear periodicity. (b) A typical noise-reduced $(m=60)$ 'isotropic' Eden cluster. (c) The angular surface anisotropy, $P(\theta)$, of the noise-reduced clusters. A clear diagonal anisotropy is present. Clusters were grown to $N=10^{5}$ elements and anisotropy values are averaged over 200 repeats.

We have explicitly tested Eden clusters grown with the relevant $\nu_{c}$ values for anisotropy. In the full model it is clear that the anisotropy of the model is reduced (c.f. Fig. 2 (a) and SM Fig. $1(\mathrm{~b}))$. No periodicity is evident and the maximum anisotropy value is well below $1 \%$ in comparison to the $\sim 2 \%$ anisotropy exhibited by the original Eden $\mathrm{C}$ model. However, the noise-reduced version of the model $(m=60)$ shows a clear positive diagonal 
anisotropy (see Figs. 2 (b) and (c)) of around $40 \%$ and a negative axial anisotropy of around $20 \%$. These anisotropies are confirmed when considering the Fourier decomposition of, $P(\theta)$ (see Section IV B of the SM). Similar results are observed for the noise-reduced version of

the 'isotropic' Eden A model (results not shown). Clearly the randomness of the order of addition of the particles is important for the 'isotropic' Eden models of Paiva and Ferreira Jr [23] to maintain isotropy. In addition to these on-lattice cluster models there have also been a variety of isotropic off-lattice versions of the Eden model [17, 20, 27], with which we shall not concern ourselves further in this on-lattice focussed manuscript.

Recently Drasdo [11] introduced an on-lattice model of cluster growth via mitosis in order to model tumor growth. An element of the cluster is chosen randomly to be a division candidate and, providing it is within distance $\Delta R$ of an unoccupied lattice site, it divides. Division is such that the two daughter elements are positioned adjacent to each other and the elements of the cluster are rearranged so that the nearest empty site to the original parent element is filled. Although it produces circular looking clusters (see Fig. 3 (a)) this model is also inherently anisotropic. Large clusters can show a periodic variation in anisotropy (indicative of an inherent anisotropy) and a peak anisotropy of about 1.5-2\% (Fig. 3 (b)). Note that this is of similar magnitude but with inverse orientation to the anisotropy found in the Eden model: in Drasdo's model the positive bias is in the diagonal directions and the negative bias in the axial directions. The dominant nature of the axial and diagonal anisotropies in Drasdo's model are confirmed by considering the Fourier decomposition of, $P(\theta)$ (see Section IV C of the SM). In the noise-reduced limit, the cluster anisotropy is even more evident [11]. It should be noted, however, that Drasdo [11] also gives an analogue of his model on a 'Dirichlet lattice' [8]. In this case there is no observable anisotropy to the clusters $[8,11]$.

\section{A MORE ISOTROPIC MODEL OF CLUSTER AGGREGATION}

We adapt the model of Ferreira Jr. and Alves [15] to include a specific particle rearrangement algorithm. This rearrangement algorithm mimics the reorganisation of cells in a biological cluster upon mitosis of an internal cell. The revised algorithm proceeds as follows: an element of the cluster is randomly selected to divide. A uniformly distributed random number (representing a clockwise angle with the $x$-axis) between 0 and $2 \pi$ (the 'direction 


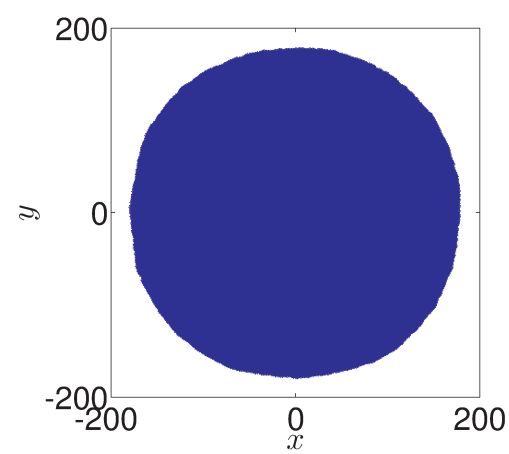

(a)

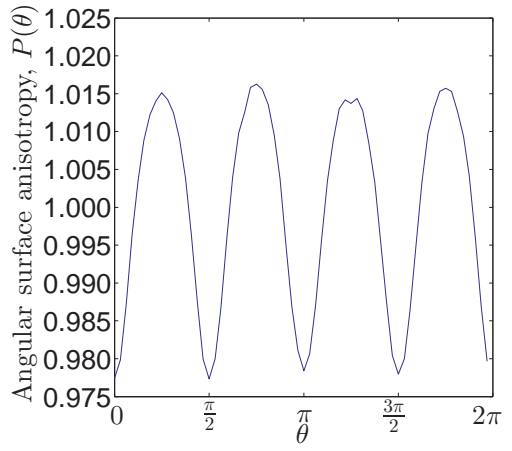

(b)

FIG. 3. The anisotropy of clusters of the Drasdo model. (a) A typical example of a cluster grown using the algorithm of Drasdo with $k=10$. The cluster displays slightly flatter edges in the axial directions. (b) The angular surface anisotropy, $P(\theta)$. Clear diagonal anisotropy of the clusters is evident. Clusters were grown to $N=10^{5}$ elements and anisotropy values are averaged over 200 repeats.

of division') is then generated (see Fig. 4(a)). Upon division a daughter element is placed on a lattice site neighboring the parent interval in the direction of division and other elements are displaced sequentially to make room. Displaced elements follow the direction of an arrow, drawn from the center of the parent element in the direction of division, to a neighboring lattice site. Elements are sequentially displaced in this way until a free lattice site is encountered (see Fig. 4(b)). The resulting model is similar to Drasdo's but is less anisotropic, even in the noise-reduced limit. In addition the specific rearrangement algorithm engenders a biological realism in the model. In particular, the algorithm can now be used to represent a discrete underlying growing domain for an on-lattice position-jump model of a reaction-diffusion process (see Section V).

Fig. 5 demonstrates the values of the anisotropy metric, $P(\theta)$, produced by this model in both the noisy and noise-reduced regimes. For a strongly anisotropic cluster we might expect to see a periodic variation in the anisotropy metric as a result of the strong contribution of a single multipole. Since, for the noisy cluster (Fig. $5(\mathrm{a})$ ), there is no clear periodicity to the values of the angular surface anisotropy metric, $P(\theta)$, we suggest that the model is more isotropic than previously proposed models of on-lattice cluster growth. We employ a Fourier decomposition of, $P(\theta)$, in Section IV D of the SM in order to investigate this suggestion further. Furthermore, for the noise-reduced variant of the model with parameter $m=60$ (see Fig. 6 (b) for a sample cluster) we find that the clusters appear even more isotropic than their random counterparts with a lower overall anisotropy of maximum absolute value 


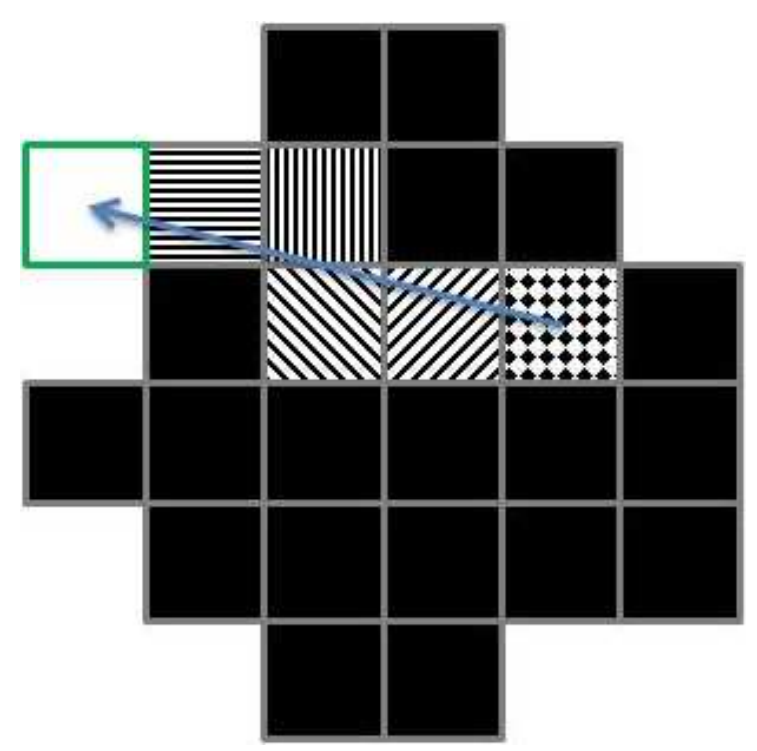

(a)

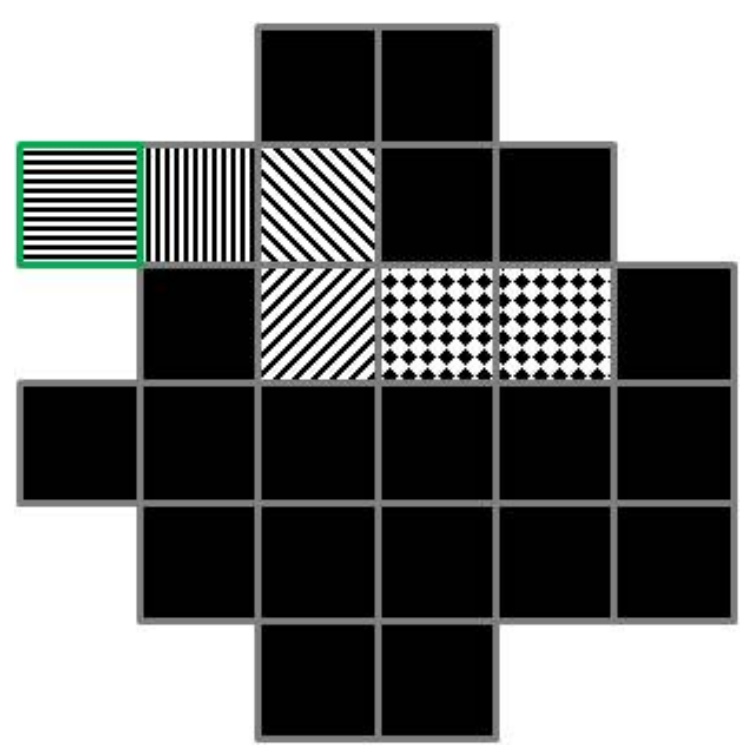

(b)

FIG. 4. The rearrangement protocol for division in a random direction from a random parent interval. (a) An element of the cluster is chosen randomly to divide (checked pattern) and a uniformly distributed random angle is used to determine the direction in which the element will divide. A line (blue arrow, $\longrightarrow$ ) is drawn from the parent interval in this direction until the first free site is encountered (green boundary, $\square$ ). (b) Elements (various patterns) are pushed in this direction in such a way that one of them occupies the free lattice site. The daughter interval (also checked) is placed on the lattice site adjacent to the original parent interval in the direction of division.

around $0.3 \%$. There do appear to be periodic variations in the anisotropy values, however, we found variations of comparable magnitude for a truly circular cluster (see SM Fig. 3 (d)). The anisotropy of the noise reduced clusters is also analysed further in Section IV D of the SM.

This model has the added benefit that it is more computationally efficient than Drasdo's since every element selected to divide produces a daughter element which increases the size of the cluster more rapidly. In Drasdo's model (especially for large cluster sizes) a large amount of time is spent selecting sites that are unable to divide because of their distance from the surface. Hence growth is retarded at long times. The slowing of growth in the model is related to saturation of the surface which allows the classification of the Drasdo model into a UC. 


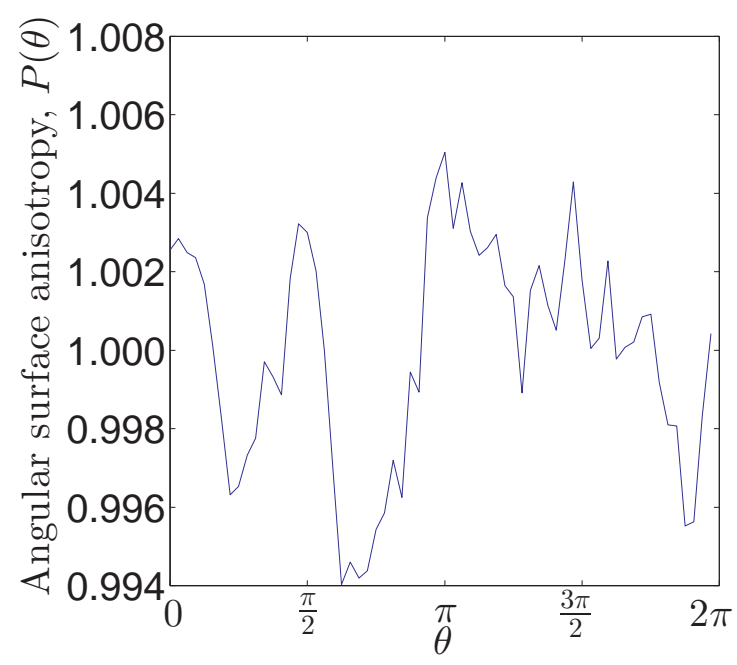

(a)

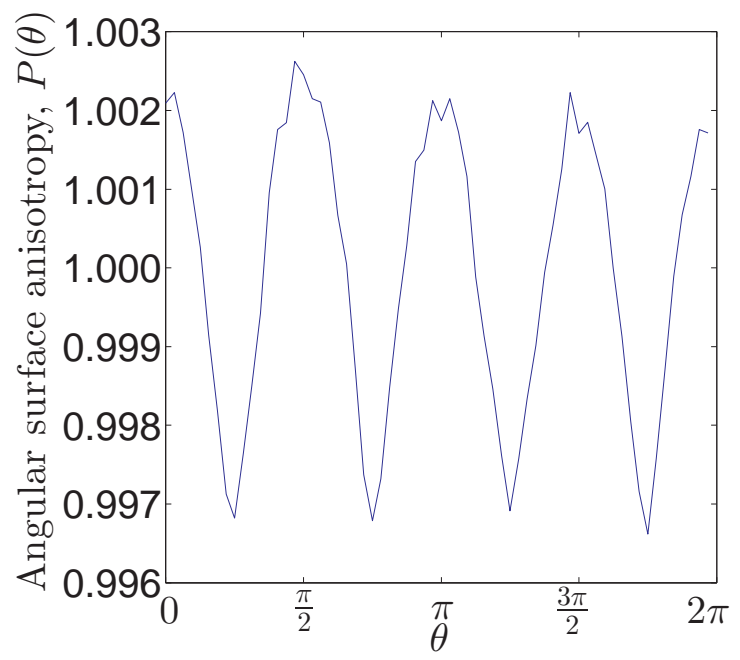

(b)

FIG. 5. The anisotropy of clusters grown by elements dividing in random directions. (a) The angular surface anisotropy, $P(\theta)$. (b) The angular surface anisotropy for the noise-reduced variant of the model with $m=60$. Clusters were grown to $N=10^{5}$ elements and anisotropy values are averaged over 200 repeats.

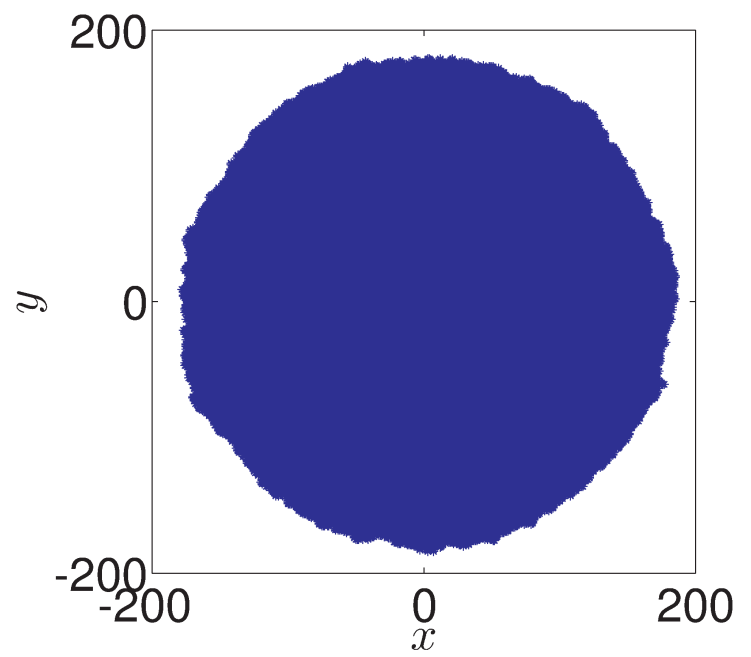

(a)

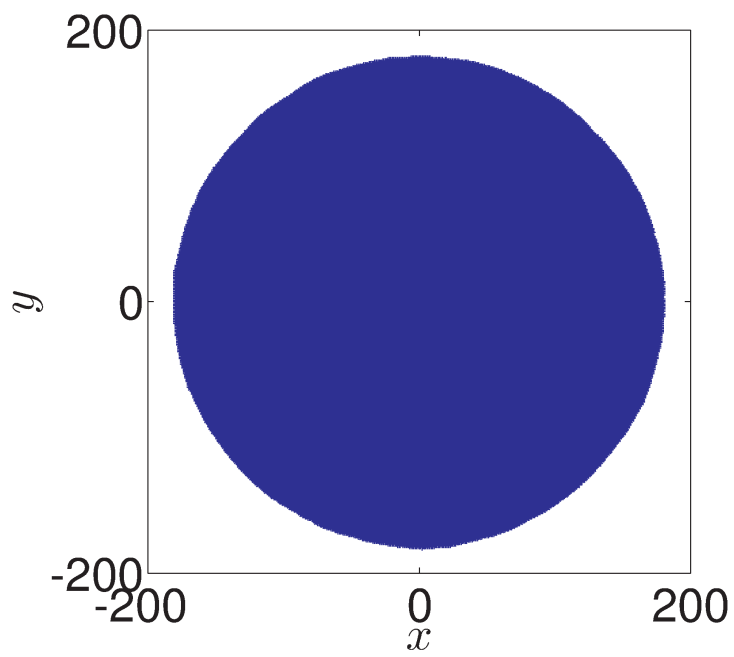

(b)

FIG. 6. Examples of clusters grown using algorithm of Ferreira Jr. and Alves [15]. (a) A typical example of such a cluster which looks relatively circular, displaying no obvious anisotropy. (b) A typical example of a cluster grown with the noise-reduced variant of the model with $m=60$. Clusters were grown to $N=10^{5}$.

\section{SURFACE SCALING AND UNIVERSALITY CLASSES}

The UC of an aggregate has traditionally been determined by considering the surface scaling properties of aggregates grown in a ' $1+1$ ' dimensional strip geometry. In the strip 
geometry clusters are grown from a base-layer of seed particles of length $l$ incorporating periodic boundary conditions on the vertical boundaries. The width of an aggregate, $w$, is defined to be some measure of the standard deviation of the height of the surface particles. For a large class of models the width is found to increase initially as a power law in the effective height, $\hat{h}=N / l$, (where $N$ is the number of particles) and then to level off to a value which is dependent on the length of the substrate upon which the aggregate is grown. The effective height at which the surface levels off can also be shown to depend, as a power law, on the length of the substrate, $l$. These relationships are summarized by the Family-Vicsek scaling relation [14]:

$$
w(l, \hat{h}) \sim l^{\alpha} f\left(\frac{\hat{h}}{l^{z}}\right), \quad f(x) \sim \begin{cases}x^{\beta}, & x \ll 1 \\ \text { constant }, & x \gg 1\end{cases}
$$

The growth exponent, $\beta$, characterizes the initial increase in surface width, whilst the roughness exponent, $\alpha$, captures the dependence of surface roughness at saturation on substrate length. The dynamic exponent $z$ determines the relationship between saturation height and substrate length. The three scaling exponents are related by $z=\alpha / \beta$. Determining these exponents enables us to classify models into UCs. Each class has its own characteristic stochastic partial differential equation of the form

$$
\frac{\partial \hat{h}(\boldsymbol{x}, t)}{\partial t}=F+\eta(\boldsymbol{x}, t)
$$

where $F$ is the average rate of particle arrival at site $\boldsymbol{x}$ and $\eta(\boldsymbol{x}, t)$ is zero-mean, uncorrelated noise which models random fluctuations in the deposition process [5]. Implicitly, by searching for the scaling coefficients which characterize a model into a particular UC, we are assuming that the surface width will eventually saturate, which, as we discover, is not necessarily a valid assumption.

Upon analyzing the model of Ferreira Jr. and Alves [15] using traditional scaling techniques, it appears to exhibit a classic power law dependence of surface width on domain length, $l$, (Fig. $7(\mathrm{a}))$ and effective height, $\hat{h}$, (Fig. $7(\mathrm{~b}))$ in the appropriate limits. This might lead us to conclude that the model follows the classic Family-Vicsek scaling relationship, equation (3). However, by studying the scaling behavior of the surface we can see 
that the surface width exhibits a much more complicated, non-monotonic dependence on the effective height. Fig. 8 (a) shows the variation of interface width with effective height for four different values of domain length. We calculate critical exponents $\alpha=0.15 \pm 0.07$ and $\beta=0.35 \pm 0.07$. When the surface width is rescaled (using the Family-Vicsek scaling relationship) by the critical exponents, derived from the scaling relationships displayed in Fig. 7, the data do not collapse onto themselves as they do for the Eden model (SM Fig. 8). This suggests a different functional form for the scaling relationship for this model.

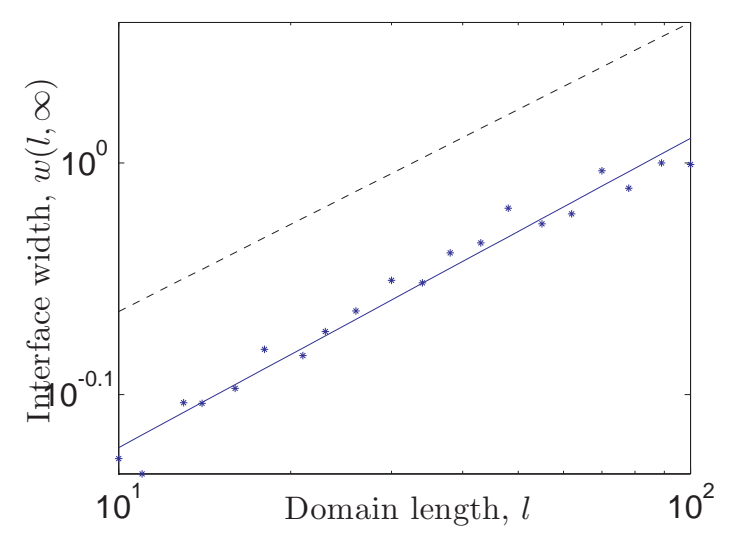

(a)

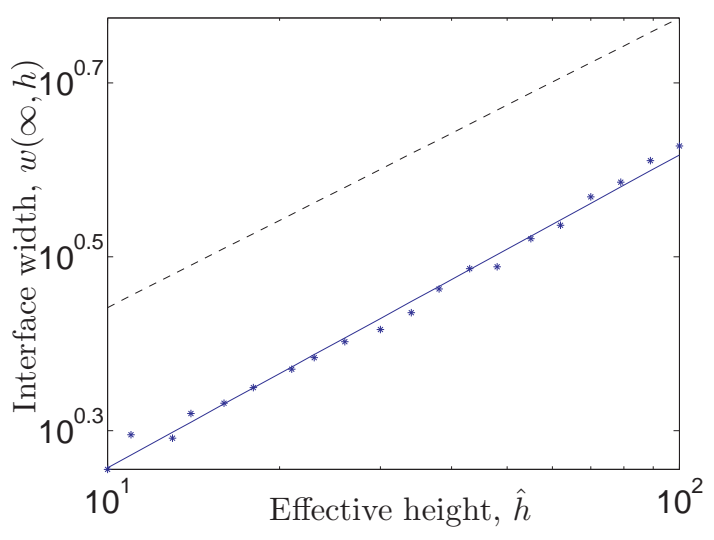

(b)

FIG. 7. Scaling of the surface width of random direction mitosis clusters in '1+1' dimensions. (a) The variation of $w(l, \infty)$ (i.e. $\hat{h} \gg l$ ) with domain length, $l$, for the random directional mitosis model. The straight (black dashed) line plotted for comparison has gradient $1 / 8$. (b) The variation of $w(\infty, \hat{h})$ (i.e. $l \gg \hat{h}$ ) with effective height, $\hat{h}$. Descriptions as for (a). The straight (black dashed) line plotted for comparison has gradient $1 / 3$.

The fact that the surface width saturates at all is an artifact of the periodic boundary conditions of the strip geometry. Division events with shallow division angles displace large numbers of elements and effectively explore the surface in search of the lowest empty lattice site to occupy. This effect introduces the surface correlations required to reduce surface width, while division events with steep division angles effectively increase the surface width. When the average surface height is low, particles chosen for division are at or near the surface a large proportion of the time. This reduces the effect of shallow division events since the surface cannot be explored fully and hence the surface width grows beyond its saturation value. As the effective height increases, a higher proportion of division events are initiated sufficiently far below the surface for shallow angle division events to allow surface exploration. This explains the non-monotonic behavior of the surface width displayed in Fig. 8 (a). In a similar division model where only elements on the bottom-most layer are 
allowed to divide (in analogy to Model I of Ferreira Jr. and Alves [15]) this non-monotonicity is not observed since the surface width cannot grow beyond its saturation value (data not shown).

The surface thickness of these aggregates no longer obeys the Family-Vicsek scaling relationship. Instead we propose that the thickness obeys a new scaling relationship:

$$
w(l, \hat{h}) \sim l^{\alpha} \hat{h}^{\beta} f\left(\frac{\hat{h}}{l^{\gamma}}\right) .
$$

In order for the scaling of the saturation thickness, $w_{\text {cap }}$, to be correct we must choose $\alpha=0.15$, the value of the roughness exponent found previously. In this case we also chose $\gamma=\alpha$ and $\beta=1$ in order to achieve a tight collapse of the data points (see Fig. $8(\mathrm{~b})$ ).

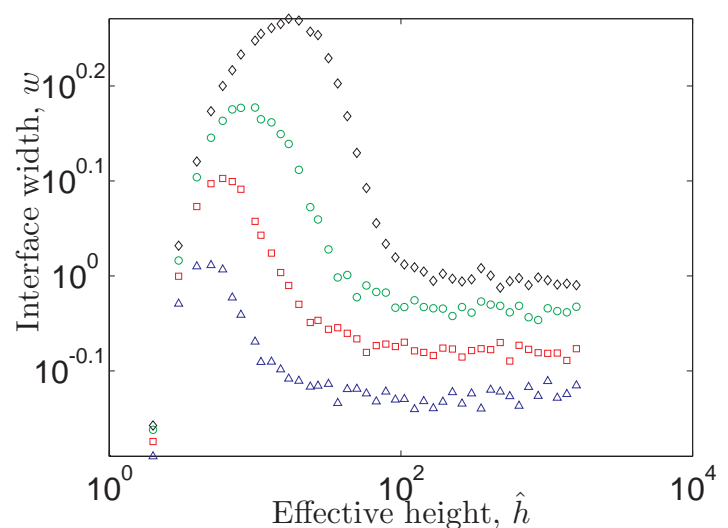

(a)

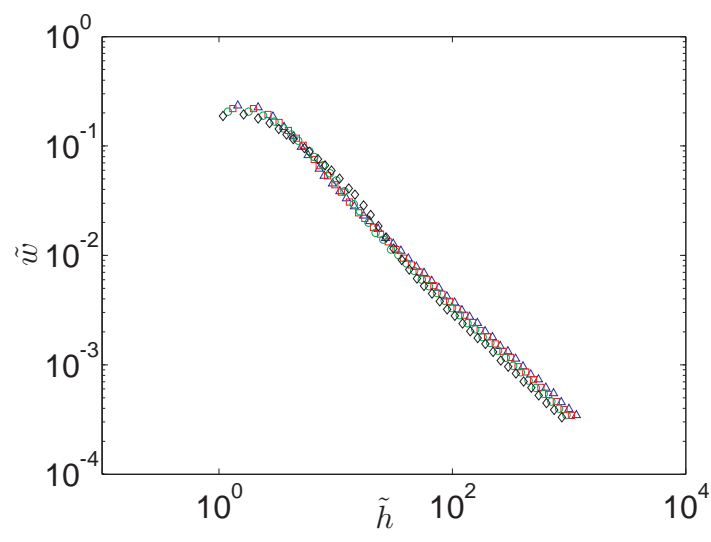

(b)

FIG. 8. The evolution of the surface width for our random direction model for different system sizes. (a) The surface exhibits an initial power law dependence on effective height, but quickly peaks, then falls and finally plateaus at the saturation value, $w_{\text {cap }}(l)$. (b) The evolution of surface width rescaled according to equation (5). Axis labels are short hand where $\tilde{w}=w(\hat{h}, l) / l^{\alpha} \hat{h}^{\beta}$ and $\tilde{h}=\hat{h} / l^{\gamma}$. A tight collapse of the data points can be seen. In both sub-figures blue triangles $(\triangle)$ correspond to system size $l=10$, red squares $(\square)$ to $l=20$, green circles $(\bigcirc)$ to $l=40$ and black diamonds ( $\diamond)$ to $l=80$.

We also investigated the surface scaling of this model in a full two-dimensional geometry. The findings of this investigation are presented visually in Fig. 9. The width is found to grow with critical exponent $\beta=0.22 \pm 0.07$ (which is in approximate agreement with the value found by Ferreira Jr. and Alves [15]). The small oscillations of the local slopes about the average value of $\beta$ in Fig. 9 (c) demonstrate that the value we have found for $\beta$ is a reliable one. This value of $\beta$ differs from the value of the growth exponent in the strip geometry, 


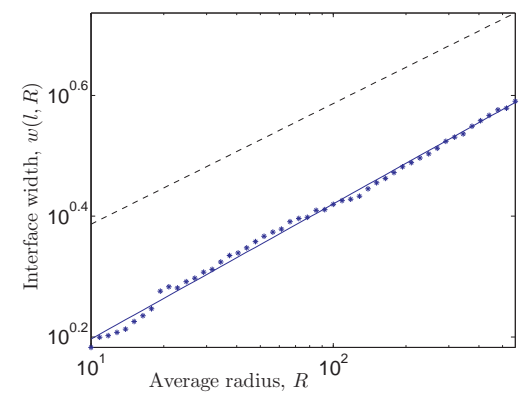

(a)

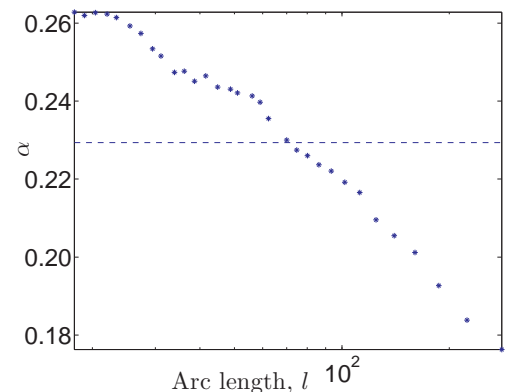

(b)

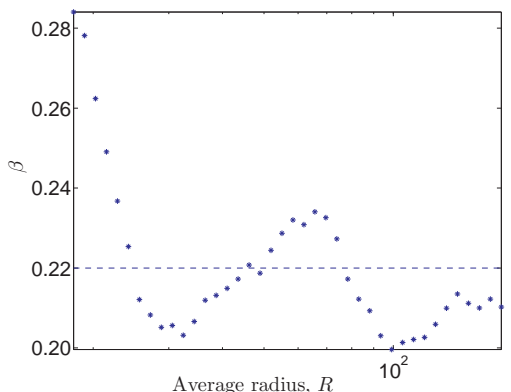

(c)

FIG. 9. Scaling of the surface width of the random direction mitosis model in two dimensions. (a) The variation of surface width, $w(l, R)$, with average cluster radius, $R$, for clusters up to $N=10^{6}$ particles. Simulations are averaged over 20 repeats. Saturation of the surface width is not evident. The straight (black dashed) line is plotted for comparison and has gradient 1/5. (b) Evaluation of the roughness exponent, $\alpha$, using the method of local gradients [5]). The mean value is plotted as a dashed blue line. (c) Evaluation of the growth exponent, $\beta$, using the method of local gradients. The mean value is plotted as a dashed blue line.

An even more stark difference between the strip geometry and the full two-dimensional geometry is the saturation behavior of the surface. In the strip geometry the surface was found to saturate with roughness coefficient $\alpha \sim 0.15$, but in the two-dimensional geometry the surface width does not appear to saturate. Fig. 9 (a) demonstrates unbounded growth of the surface width for clusters of up to $N=10^{6}$ particles. We should expect the surface width not to saturate since additions to the surface are completely uncorrelated. A consistent value for the roughness exponent, $\alpha$, is unobtainable (see Fig. 9 (b)). Since the critical exponent describes the scaling of the surface thickness with a local length scale at saturation, $w_{\text {cap }} \sim l^{\alpha}$, the inability to determine $\alpha$ is consistent with our hypothesis that the surface width does not saturate.

\section{COMPARISON OF REACTION-DIFFUSION MODELS ON A GROWING LATTICE}

Although the traditional methods of analysing and classifying surface scaling are not sufficient for the model of Ferreira Jr. and Alves [15], this does not prevent us from utilizing the isotropic properties of the model. After the introduction a specific element rearrangement algorithm in Section III we can now employ the domain cluster aggregation model 
of Ferreira Jr. and Alves [15] in order to simulate discrete, stochastic reaction-diffusion processes on a radially growing domain in two dimensions. This can be quantitatively compared to an equivalent continuum model for the same process on a deterministically growing domain. This is a natural extension of the work, in one dimension, of Baker et al. [4] and yates2012gfm in to higher dimensions. The simulations might mimic a biological tissue (a tumor for example) which is growing radially and upon which a reaction-diffusion process is taking place [24].

For demonstrative purposes we will consider a purely diffusive process in the current work, but note that the incorporation of reactions or an external signalling profile to which particles can respond would be straightforward $[4,18,22]$. We allow particles to diffuse on an initially (approximately) circular domain tessellated with a square lattice. The domain grows as described in Section III with an element being chosen to divide from all the currently available elements with equal probability at each time-step [2]. In addition, when an element divides the particles that originally resided in the parent element are split roughly evenly between the two daughter elements using the binomial distribution [4]. The elements that move in order to make room for the daughter elements do so according to the cluster rearrangement algorithm outlined in Section III and take the particles at their lattice site with them, causing an advective flow of particles due to the domain growth. We restrict particles in the individual-level model to remain in the domain which corresponds to a zero flux boundary condition in the equivalent the continuum model.

Given the constant rate of element splitting, $\varrho$, in the individual-level model, we can write down a probability master equation for the evolution of the number of domain elements at time $t$. This master equation determines that the number of domain elements grows exponentially. This, coupled with the fact that each element is equally likely to split and that the growth model is isotropic, leads us to assume uniformly exponential domain growth in the continuum model. The growth occurs at a rate, $\rho$, which we can easily relate to the splitting rate, $\varrho$, in the discrete model: the increase in area due to a division event must produce a corresponding increase in area in the continuum model and (since growth occurs isotropically) this corresponds to an increase in radius in the continuum model.

Since the PDE, boundary and initial conditions are circularly symmetric the populationlevel solution will be circularly symmetric for all time. This means we need only solve a 
one-dimensional PDE for population density, $u(r, t)$, in the continuum model:

$$
\frac{\partial u}{\partial t}+\frac{1}{r} \frac{\partial}{\partial r}(r v u)=D\left(\frac{\partial^{2} u}{\partial r^{2}}+\frac{1}{r} \frac{\partial u}{\partial r}\right) \quad \text { for } \quad r \in[0, \exp (\rho t) / 2], \quad t \in[0, \infty)
$$

The flow due to domain growth is given by $v=d r / d t=\rho r$, where $\rho$ is the strain, which is determined from the element splitting rate, $\varrho$, in the individual-level model. We chose the initial domain to have radius $1 / 2$ which, therefore, determines the size of each lattice element in the individual-based model. We solve equation (6) using the NAG solver D03PE $[25]$.

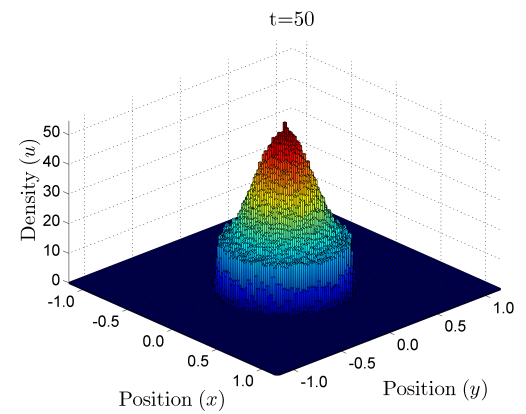

(a)

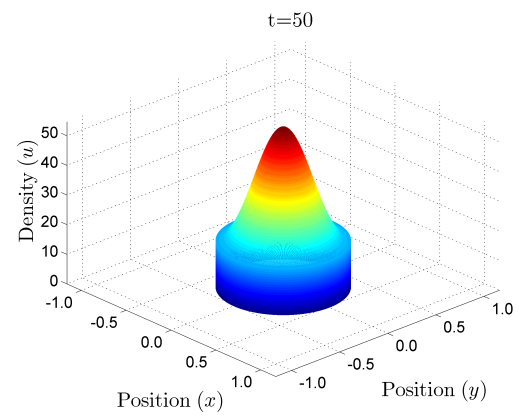

(b)

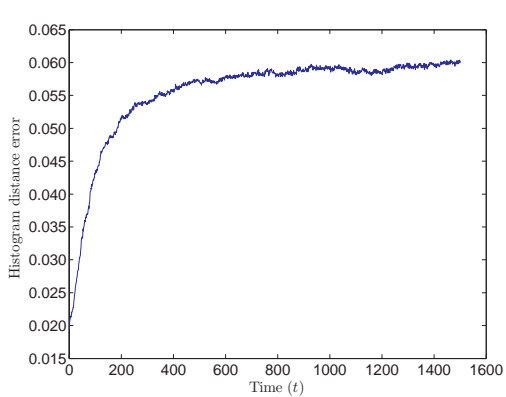

(c)

FIG. 10. A comparison of the individual-level (with square domain tessellation) and population-level models of diffusion on a growing circular domain. In the individual-level model 50, 000 particles are initialised with a density profile which decays approximately exponentially from the centre to the edge of the domain. The continuum model is initialised similarly with a profile $u=A \exp (-10 r)$, where $A$, the normalising factor, ensures that the area under the continuum initial condition corresponds to the correct number of particles in the individual-level simulation. There are initially 1885 elements in the circular domain of the individual-level model. Over a simulation time of 1500 time units, with an element splitting rate of $\varrho=0.001$, this number grows to $\sim 8448$. (a) The histograms represent the density of particles in each square of the domain tessellation. (b) The surface represents the radially symmetric solution of $P D E(6)$ with $D=h^{2}$, where $h=1 / 49$ is the length of an element in the individual-level model. (c) The evolution of the histogram distance error (HDE) (see Section VI of the SM for a definition of the HDE metric).

A snapshot comparison of the densities of the two models is given in Fig. 10. The models appear to give qualitatively similar results. We also plot the evolution of the histogram distance error (HDE) in Fig. 10 (c). The HDE, which gives an indication of how closely the two models correspond (see Section VI of the SM for a definition of the HDE metric), is low, indicating a good quantitative comparison between the individual-level and population-level domain growth models of diffusion on a radially growing domain. 


\section{DISCUSSION}

In summary, we demonstrated that that the model of Ferreira Jr. and Alves [15] is capable of growing circular clusters free from the inherent anisotropy of the underlying square lattice. Our revised model description also includes an explicit algorithm for rearrangement of the cluster upon division of an element. Such an isotropic model will be essential when modeling domain growth in biological contexts where no anisotropy is present. As a representative example we presented a comparison between an individual-level, position-jump model of particle diffusion on a radially growing domain and a continuum counterpart.

Our investigation into the surface scaling properties of clusters generated by the domain growth model suggests that the traditional mechanisms for classifying growing aggregates into UCs, based on the saturation behavior of their surface widths (often in a strip geometry), may be inadequate for partitioning models, such as that of Ferreira Jr. and Alves [15], whose clusters do not grow through particle additions whose probabilities are based purely on surface considerations. In particular, the model for isotropic cluster growth analysed in this manuscript does not exhibit Family-Vicsek scaling behavior and, as such, cannot be placed in a traditional UC. Further work is required to determine whether the inability to be classified into UCs is common to other aggregation models whose particles are added using more global considerations.

\section{ACKNOWLEDGMENTS}

CAY would like to thank Christ Church College for a Junior Research Fellowship.

[1] . Choosing $K$ to be a sufficiently large power of two ensures that each axis and diagonal has a sector of the circle centered upon it. This ensures that we measure the anisotropy in each of these directions in a consistent manner. In all the anisotropy measurements presented in this paper we choose $K=64$.

[2] . By choosing a single particle per evenly spaced time-step we will generate exponential domain growth. However, we note that by altering the rate at which a particle are chosen we can incorporate different types of domain growth including linear and logistic, for example. 
[3] S.G. Alves and S.C. Ferreira Jr. Is it really possible to grow isotropic on-lattice diffusionlimited aggregates? J. Phys. A.-Math. Gen., 39:2843, 2006.

[4] R.E. Baker, C.A. Yates, and R. Erban. From microscopic to macroscopic descriptions of cell migration on growing domains. Bull. Math. Biol., 72(3):719-762, 2010.

[5] A.L. Barabási and H.E. Stanley. Fractal Concepts in Surface Growth. Cambridge University Press, 1995.

[6] M.T. Batchelor and B.I. Henry. Limits to Eden growth in two and three dimensions. Phys. Lett. A, 157(4-5):229-236, 1991.

[7] M.T. Batchelor, B.I. Henry, and S.D. Watt. Continuum model for radial interface growth. Physica A, 260(1-2):11-19, 1998.

[8] M. Block, E. Schöll, and D. Drasdo. Classifying the expansion kinetics and critical surface dynamics of growing cell populations. Phys. Rev. Lett., 99(24):248101, 2007.

[9] V.A. Bogoyavlenskiy. How to grow isotropic on-lattice diffusion-limited aggregates. J. Phys. A.-Math. Gen., 35:2533, 2002.

[10] T. Callaghan, E. Khain, L.M. Sander, and R.M. Ziff. A stochastic model for wound healing. J. Stat. Phys., 122(5):909-924, 2006.

[11] D. Drasdo. Coarse graining in simulated cell populations. Adv. Complex. Syst., 8(3):319, 2005.

[12] M. Eden. A Probabilistic Model for Morphogenesis, chapter 4: Information Networks, pages 359-370. Pergamon Press, New York, 1958.

[13] M. Eden. A two-dimensional growth process. In Proceedings of the Fourth Berkeley Symposium on Mathematics Statistics and Probability, volume 4, pages 223-239. University of California Press, 1961.

[14] F. Family and T. Vicsek. Scaling of the active zone in the Eden process on percolation networks and the ballistic deposition model. J. Phys. A.-Math. Gen., 18(2):L75-L81, 1985.

[15] S.C. Ferreira Jr. and S.G. Alves. Pitfalls in the determination of the universality class of radial clusters. J. Stat. Mech.-Theory. E., 2006(11):P11007, 2006.

[16] P. Freche, D. Stauffer, and H.E. Stanley. Surface structure and anisotropy of Eden clusters. J. Phys. A.-Math. Gen., 18:L1163-L1168, 1985.

[17] P.F. Ho and C.Y. Wang. Cluster growth by mitosis. Math. Biosci., 155(2):139-146, 1999.

[18] J. Hu, H.-W. Kang, and H.G. Othmer. Stochastic analysis of reaction-diffusion processes. Bull. Math. Biol., 2013. 
[19] R. Jullien and R. Botet. Surface thickness in the Eden model. Phys. Rev. Lett., 54(18): 2055-2055, 1985.

[20] R. Jullien and R. Botet. Aggregation and Fractal Aggregates. World Scientific, Singapore, 1987.

[21] P. Meakin. Universality, nonuniversality, and the effects of anisotropy on diffusion-limited aggregation. Phys. Rev. A, 33(5):3371-3382, 1986.

[22] H.G. Othmer, S.R. Dunbar, and W. Alt. Models of dispersal in biological systems. J. Math. Biol., 26(3):263-298, 1988.

[23] L.R. Paiva and S.C. Ferreira Jr. Universality class of isotropic on-lattice Eden clusters. J. Phys. A.-Math. Theor., 40(1):F43-F49, 2007.

[24] A.M. Smith, R.E. Baker, D. Kay, and P.K. Maini. Incorporating chemical signalling factors into cell-based models of growing epithelial tissues. J. Math. Biol., 2011.

[25] The Numerical Algorithms Group. NAG Toolbox for Matlab, 2009.

[26] G. Wagner, R. Halvorsrud, and P. Meakin. Extended Eden model reproduces growth of an acellular slime mold. Phys. Rev. E, 60(5):5879-5887, 1999.

[27] C.Y. Wang, P.L. Liu, and J.B. Bassingthwaighte. Off-lattice Eden-C cluster growth model. J. Phys. A.-Math. Gen., 28(8):2141-2147, 1995. 